\section{CHILDHOOD IMMUNISATION - SO NEAR AND YET SO FAR}

\section{GUEST EDITORIAL}

Mark J Ferson.

Director, Public Health Unit

South Eastern Sydney Area Health Service

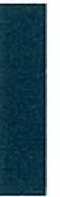

$\mathrm{n}$ this 200th anniversary year of Jenner's observation that inoculation with cowpox protected against smallpox, it is timely to review the status of childhood immunisation in NSW. Considerable effort is made by staff based in Public Health Units and community health services to maintain surveillance of immunisation uptake among young children. Although a vocal minority attests otherwise, immunisation remains a very cost-effective and safe public health intervention, providing benefit to the individual as well as to the community.

The history of immunisation in Australia goes back to 1804, less than a decade after Jenner's discovery, when packets of smallpox vaccine arrived in Sydney Town for use in infants. Mass programs as we now understand them were instituted during the 1940 s to provide protection against diphtheria, tetanus and pertussis (whooping cough). Live viral vaccines were added to the schedule later, oral polio vaccine (Sabin) in 1966 and rubella vaccine for schoolgirls in 1971. In the space of little over one generation, we have seen the effects of immunisation - poliomyelitis and diphtheria have been eradicated, the incidence of tetanus has fallen remarkably, and congenital rubella infection is rare. The addition of conjugated vaccines against Haemophilus influenzae type $\mathrm{b}$ (Hib) to the publicly funded infant vaccination program in 1993 has resulted in more than a 90 per cent decline in the incidence of life-threatening infections caused by this organism.

Despite these enormous successes, substantial challenges remain. The incidence rates of measles and pertussis have undoubtedly declined since the introduction of vaccines. However, these highly infectious diseases require very high levels of 'herd immunity' (immunity in a population) to break the chain of transmission. Our immunisation processes have not achieved control of either measles or pertussis, although there has not yet been time for recent changes to the schedule to take effect (a fifth dose of pertussis vaccine, given before entry to school, and a second dose of measles-mumps-rubella vaccine, given to boys and girls in high school). In 1993 (when the last epidemic peak for both infections occurred), 2,397

\section{Contents}

\section{Articles}

111 Cbildbood immunisation - so near and yet so far. Guest editorial

113 Public Health Network report on immunisation

117 Immunisation status of two-yearold cbildren in the Hunter Area

118 Long day care centre immunisation rates in Western Sydney

120 Issues in administration of the immunisation provisions of the Public Health Act 1991

122 A review of school entry immunisation certificates in the Manly, Warringab and Pittwater area, 1995

124 School entry immunisation certificates in South Western Sydney, 1995

126. Infectious Diseases

\section{Correspondence}

Please address all correspondence and potential contributions to:

The Editor;

NSW Public Health Bulletin, Public Health Division, NSW Health Department Locked Bag No 961, Nortb Sydney NSW 2059 Telephone: (O2) 93919191 Facsimile: (02) 93919029 


\section{Childhood immunisation editorial}

Continued from page 111

cases of measles and 1,546 cases of pertussis were notified in NSW, while 603 measles and 1,389 pertussis cases were notified in 1995.

So what has been done and what remains to be done? The Australian Bureau of Statistics survey, Children's Immunisation Australia, conducted in April 1995, found that only 54 per cent of NSW children aged three months to six years were fully immunised for their age (Hib vaccines were excluded)'. Although the validity of these data is open to question because the survey relied on parental recall, it is known that immunisation uptake falls markedly in the second year of life ${ }^{2}$. This is probably due more to the pressures of life within young families and other barriers, than to parents disagreeing with the need for vaccination. Lack of interest, and confusion about the schedule or about vaccine contraindications among some providers, are also contributory factors ${ }^{3,4}$ as is the lack of acceptance of the principle of opportunistic immunisation ${ }^{5}$.

This issue of the Public Health Bulletin reports on three studies which used the immunisation provisions of the Public Health Act 1991 to examine immunisation compliance among NSW children. The Public Health Act 1991 was amended in 1992 with provisions relating to immunisation of children attending child-care facilities (long day child-care, preschool, family day care or a registered playgroup) and primary schools. These provisions, which came into effect in 1994, place the onus on directors of child-care facilities and school principals to record the immunisation status of children in their institutions. In addition, the provisions empower the Medical Officer of Health of the local Public Health Unit to direct that healthy but unimmunised children in contact with a case be excluded from the child-care facility or school. The rationale for the amendments was to remind parents to have their children fully immunised. It was envisaged that proper documentation of immunisation would also help in controlling, for example, an outbreak of measles in a school or preschool by simplifying identification of unimmunised contacts. At the same time the recording of immunisation status can provide a valuable means of surveillance of immunisation coverage.

A study of immunisation coverage among two-year-old children attending child care in the Hunter Area (page 117), and the Western Sydney and Wentworth Areas (page 118) suggested that the majority of long day care centres were able to provide information on immunisation status. However, in metropolitan areas at least, it was not feasible for family day care schemes to collect this information. The authors observed that information from records of immunisation status in child-care facilities does not provide a reliable indicator of immunisation coverage at two years of age, which is a key index of child health promoted by the World Health Organisation. An alternative approach is required, and the Australian Childhood Immunisation Register (ACIR) has the potential to fill this important gap.

Studies carried out in primary schools in Sydney's northern beach suburbs (page 122), South Western Sydney (page 124) and the former Eastern Sydney Area (page 120) used different methods to assess immunisation compliance in kindergarten and year 1 students by review of the official immunisation certificates. Estimates of immunisation compliance varied widely between the three studies; for example, the minimal estimates of the proportion of kindergarten children fully immunised ranged from 45 per cent on the northern beaches to 77 per cent in Eastern Sydney. However, common themes emerged: many children did not provide immunisation certificates; a high proportion of those provided were incorrectly filled out; school staff were often unable to interpret the certificates or other immunisation documents; and in some cases providers needed several reminders to issue certificates to the parents. These authors considered the immunisation provisions of the Public Health Act 1991 would make an important contribution to the improvement of immunisation rates in NSW children. However, there was consensus that school staff and immunisation providers needed much assistance in making the system work effectively.

As a consequence of the introduction of new vaccines and changes in disease epidemiology induced by mass vaccination, the childhood immunisation program is subject to constant change. This causes difficulties for parents and immunisation providers, as well as for public health personnel who must keep the community informed of new developments. One clear message is that vaccines (for example, those against hepatitis $B$ and varicella) must not be added to the routine schedule until combination vaccines become available.

The establishment of the ACIR provides the first opportunity to enlist all immunisation providers and Australian children into a unified system. Once inevitable teething problems with participation and data collection are overcome, the ACIR will be able to generate valuable detailed information on immunisation coverage in any group defined by age or geography.

Despite extensive epidemiological research on childhood immunisation, further research must be encouraged and supported to fill major gaps in knowledge. Little is known about the efficacy of some of our current vaccines 


\section{DISCUSSION}

In this study of immunisation rates in children aged 2-3 years enrolled in LDCCs in greater western Sydney, the up-to-date immunisation rates at two years of age for three doses of DTP, four doses of DTP, three doses of OPV, one dose of MMR and all eight immunisations were 94 per cent, 78 per cent, 92 per cent, 91 per cent and 76 per cent respectively. There is a precipitous drop in the rate of immunisation with the fourth dose of DTP. Reasons for nonimmunisation with the fourth dose of DTP were not elicited, but need to be examined. Not unexpectedly, immunisation rates at the time of the survey were higher compared to immunisation rates at two years of age.

The National Immunisation Strategy (NIS) ${ }^{6}$ aims to achieve $>90$ per cent coverage of children aged two years for all diseases specified in the childhood immunisation schedule by 2000 . In this survey of LDCCs, NIS targets for three doses of DTP, three doses of OPV and one dose of MMR were achieved, but immunisation rates for four doses of DTP and hence for all eight immunisations were substantially lower than the 2000 target. This is of concern since we would expect children attending LDCCs to have higher immunisation rates than non-attenders?.

Immunisation registers in LDCCs in NSW are used as surveillance tools. However, only about 14 per cent of all children $<4$ years of age in NSW attend $\mathrm{LDCCs}^{8,9}$. The immunisation status of these children may be quite different from children who do not attend LDCCs, and we should be cautious when generalising such results to children who do not attend LDCCs. Preschools may provide an additional source of surveillance information which may complement data from LDCCs.

We presented two sets of immunisation rates (including up-to-date rates) in this study. Whether immunisation rates should be presented as age-appropriate rates (not presented here) or as up-to-date rates is debated ${ }^{10,11}$. There are also views on how age-appropriate immunisation rates should be calculated. Standard definitions for age-appropriate and up-to-date immunisation rates should be developed to allow immunisation rates within and between States and Territories to be compared.

The low rates for four doses of DTP and all eight immunisations at the age of two years indicate that we need to target groups identified to have poor immunisation rates. We need to understand reasons for non-immunisation and delayed immunisation, and instigate innovative programs to ensure that more children - especially high-risk children are immunised, and at the recommended age, to enable us to achieve NIS targets.

\section{ACKNOWLEDGEMENTS}

We thank all the directors and staff of the long day care centres who participated in this study. We also thank Susan DeBrincat-Trapuzzano for data entry, and the NSW Health Department for the SSISS methodology.

1. NSW Immunisation Requirements for school entry from 1994, State Health Publication Number (EB) 92-87, ISBN 0730535975. 2. Klein JO. Infectious diseases and day care. Review of Infectious Diseases 1986; 8(4):521-6.

3. Thacker SB, Addiss DG, Goodman RA et al. Infectious diseases and injuries in child day care. Opportunities for healthier children. Journal of the American Medical Association 1992; 268(13):1720-6.

4. Microsoft Access. Relational database management system for windows, Version 2.0. Microsoft Corporation 1994.

5. SAS Institute Inc. SAS ${ }^{3}$ Procedures Guide, Release 6.03 Edition. Cary, NC: SAS Institute Inc, $1988.441 \mathrm{pp}$.

6. National Immunisation Strategy. Report by the Panel on a National Immunisation Strategy on behalf of the Communicable Diseases Standing Committee. NHMRC April 1993. Commonwealth of Standing Commitralia 1998

7. Hinman AR. Vaccine-preventable diseases and child day care. Review of Infectious Diseases $1986 ; 8(4): 573-83$.

8. 1991 Census of Child Care Services Summary. Department of Human Services and Health. Commonwealth of Australia 1994. Australian Government Publishing Service, Canberra. ISBN 0644354801.

9. Australian Bureau of Statistics. 1991 Demography New South Wales. Commonwealth of Australia. Catalogue No 3311.1.

Commonwealth of Australia. Catalogue No 3311.1 .
10. Varrasso DA, Redlener I. Defining delayed immunisation. (Letter) 10. Varrasso DA, Redlener I. Defining delayed im
Pediatric Infectious Disease Journal 1992;11:897.

11. Dietz DJ, Zell ER, Stevenson J. Defining delayed immunisation. (Letter) Pediatric Infectious Disease Iournal 1993; 12(4):353-354.

\section{Childhood immunisation editorial}

$\checkmark$ Continued from page 112

or about age-specific susceptibility to the major vaccinepreventable diseases. Until this information is available, and is used to tailor the childhood (and adult) immunisation program, control of highly infectious diseases such as measles and pertussis is but a pipedream.
1. Australian Bureau of Statistics. Children's immunisation, Australia, April 1995. Canberra: ABS, 1996. Catalogue no. 4352.0.

2. Herceg A, Daley C, Schubert P, Hall R, Longbottom $H$. A populationbased survey of immunisation coverage in two-year-old children. Aust $J$ Public Health 1995; 19:645-470.

3. Ferson MJ, Christie D. True and false contraindications to pertussis 3. Ferson MJ, Christie D. True and false
vaccine. Med J Aust 1990; 153:568-569.

4. Rixon G, March L, Holt DA. Immunisation practices of general practitioners in metropolitan Sydney. Aust J Public Health 1994; $18: 258-260$

5. Burgess MA, Levy M, Alperstein G et al. 'On the spot' vaccination: does it work? I Paediatr Child Health 1996; 32:63-67. 